Bull. Korean Math. Soc. 49 (2012), No. 4, pp. 815-827

http://dx.doi.org/10.4134/BKMS.2012.49.4.815

\title{
EXISTENCE OF $n$ POSITIVE SOLUTIONS TO SECOND-ORDER MULTI-POINT BOUNDARY VALUE PROBLEM AT RESONANCE
}

\author{
Feng Wang and Fang Zhang
}

\begin{abstract}
The existence of $n$ positive solutions is established for secondorder multi-point boundary value problem at resonance where $n$ is an arbitrary natural number. The proof is based on a theory of fixed point index for A-proper semilinear operators defined on cones due to Cremins.
\end{abstract}

\section{Introduction}

In this paper, we are concerned the multi-point boundary value problem (BVP for short)

$$
\left\{\begin{array}{l}
-x^{\prime \prime}(t)=f(t, x), \quad t \in(0,1), \\
x(0)=\sum_{i=1}^{m-2} \alpha_{i} x\left(\xi_{i}\right), \quad x(1)=\sum_{i=1}^{m-2} \beta_{i} x\left(\xi_{i}\right),
\end{array}\right.
$$

where $f:[0,1] \times \mathbb{R} \rightarrow \mathbb{R}$ is continuous, $m>2,0<\xi_{1}<\xi_{2}<\cdots<\xi_{m-2}<$ $1, \alpha_{i}, \beta_{i} \geq 0, i=1,2, \ldots, m-2$ with condition

$$
\sum_{i=1}^{m-2} \alpha_{i}=\sum_{i=1}^{m-2} \beta_{i}=1
$$

We note that (1.2) means that the multi-point boundary value problem (1.1) happens to be at resonance in the sense that the associated linear homogeneous boundary value problem

$$
\left\{\begin{array}{l}
-x^{\prime \prime}(t)=0, \quad t \in(0,1) \\
x(0)=\sum_{i=1}^{m-2} \alpha_{i} x\left(\xi_{i}\right), \quad x(1)=\sum_{i=1}^{m-2} \beta_{i} x\left(\xi_{i}\right)
\end{array}\right.
$$

has $x(t) \equiv c, t \in[0,1], c \in \mathbb{R}$, as a nontrivial solution.

Received April 20, 2011; Revised March 22, 2012.

2010 Mathematics Subject Classification. 34B10, 34B15.

Key words and phrases. boundary value problem, positive solution, resonance, A-proper, fixed point index.

The project is supported financially by the National Natural Science Foundation of China (11171090, 10971179), the Program for New Century Excellent Talents in University (NCET10-0325) and the Natural Science Foundation of Changzhou University (JS201008). 
Motivated by the work of Bitsadze and Samarskii on nonlocal linear elliptic boundary value problem [2,3], Il'in and Moiseev studied a multi-point boundary value problems for linear second-order ordinary differential equations [13]. Since then, great efforts have been devoted to the multi-point boundary value problems for more general nonlinear ordinary differential equations due to its theoretical challenge and its great potential applications; see for example $[1,6,8,9,10,11,12,14,15,16,17,18,19,21,22,25,30,31,32,33,34,35$, $36,37,38,39]$ and the references therein. Some theorems and methods of nonlinear functional analysis have been applied to research on this problem, such as the method of topological degree and Leray-Schauder fixed point theorem $[15,30,31,33,34,37]$, the upper and lower solutions method and monotone iterative technique [38], and Mawhin coincidence degree theory [21, 22]. Boundary value problems at resonance in the continuous setting have been studied by several authors, see $[6,8,9,19]$ for some references along this line. In most real problems, only the positive solution is significant. It is well known that the problem of existence of positive solutions to boundary value problem is very difficult when the resonant case is considered. To our best knowledge, only few papers deal with positive solutions to boundary value problems at resonance $[1,12,14,16,25,32,35,36,39]$. Recently, Yang and Shen [36] obtained the existence of a positive solution for problem (1.1) with resonant condition (1.2) or

$\sum_{i=1}^{m-2} \alpha_{i} \neq 1, \sum_{i=1}^{m-2} \beta_{i} \neq 1, \sum_{i=1}^{m-2} \alpha_{i} \xi_{i}\left(1-\sum_{i=1}^{m-2} \beta_{i}\right)+\left(1-\sum_{i=1}^{m-2} \alpha_{i}\right)\left(1-\sum_{i=1}^{m-2} \beta_{i} \xi_{i}\right)=0$.

The main method is the Leggett-Williams norm-type theorem due to O'Regan and Zima [24]. But there are no results concerning with the multiplicity of positive solutions for (1.1). Inspired by the above work, the aim of this paper is to establish the existence results of $n$ positive solutions for boundary value problem (1.1), where $n$ is an arbitrary natural number. This will be done by applying the theory of a fixed point index for A-proper semilinear operators defined on cones obtained by Cremins [4].

The remaining part of the paper is organized as follows. In Section 2, some preliminaries and lemmas will be given, and the main theorems are formulated and proved in Section 3. Finally, in Section 4, we give an example to illustrate our results.

\section{Notation and preliminaries}

We start by introducing some basic notation relative to theory of the fixed point index for A-proper semilinear operators defined on cones established by Cremins (see [4]).

Let $X$ and $Y$ be Banach spaces, $D$ a linear subspace of $X,\left\{X_{n}\right\} \subset D$, and $\left\{Y_{n}\right\} \subset Y$ sequences of oriented finite dimensional subspaces such that $Q_{n} y \rightarrow y$ 
in $Y$ for every $y$ and $\operatorname{dist}\left(x, X_{n}\right) \rightarrow 0$ for every $x \in D$ where $Q_{n}: Y \rightarrow Y_{n}$ and $P_{n}: X \rightarrow X_{n}$ are sequences of continuous linear projections. The projection scheme $\Gamma=\left\{X_{n}, Y_{n}, P_{n}, Q_{n}\right\}$ is then said to be admissible for maps from $D \subset X$ to $Y$.

Definition 2.1 ([4]). A map $T: D \subset X \rightarrow Y$ is called approximation-proper (abbreviated A-proper) at a point $y \in Y$ with respect to $\Gamma$, if $\left.T_{n} \equiv Q_{n} T\right|_{D \cap X_{n}}$ is continuous for each $n \in \mathbb{N}$ and whenever $\left\{x_{n_{j}}: x_{n_{j}} \in D \cap X_{n_{j}}\right\}$ is bounded with $T_{n_{j}} x_{n_{j}} \rightarrow y$, then there exists a subsequence $\left\{x_{n_{j_{k}}}\right\}$ such that $x_{n_{j_{k}}} \rightarrow x \in D$, and $T x=y . T$ is said to be A-proper on a set $D$ if it is A-proper at all points of $D$.

Let $K$ be a cone in a finite dimensional Banach space $X$ and $\Omega \subset X$ be open and bounded with $\Omega \cap K=\Omega_{K} \neq \emptyset$. Let $T: \bar{\Omega}_{K} \rightarrow K$ be continuous such that $T x \neq x$ on $\partial \Omega_{K}=\partial \Omega \cap K$ where $\bar{\Omega}_{K}$ and $\partial \Omega_{K}$ denote the closure and boundary, respectively, of $\Omega_{K}$ relative $K$. Let $\rho: X \rightarrow K$ be an arbitrary retraction.

The following definition of finite dimensional index forms the basis of generalized index for A-proper maps $I-T$.

Definition 2.2 ([4]). We define

$$
i_{K}(T, \Omega)=\operatorname{deg}_{B}\left(I-T \rho, \rho^{-1}(\Omega) \cap B_{R}, 0\right),
$$

where the degree is the Brouwer degree and $B_{R}$ is a ball containing $\Omega_{K}$.

Now let $K$ be a cone in an infinite dimensional Banach space $X$ with projection scheme $\Gamma$ such that $Q_{n}(K) \subseteq K$ for every $n \in \mathbb{N}$. Let $\rho: X \rightarrow K$ be an arbitrary retraction and $\Omega \subset X$ an open bounded set such that $\Omega_{K}=$ $\Omega \cap K \neq \emptyset$. Let $T: \bar{\Omega}_{K} \rightarrow K$ be such that $I-T$ is A-proper at 0 . Write $K_{n}=K \cap X_{n}=Q_{n} K$ and $\Omega_{n}=\Omega_{K} \cap X_{n}$. Then $Q_{n} \rho: X_{n} \rightarrow K_{n}$ is a finite dimensional retraction.

Definition 2.3 ([4]). If $T x \neq x$ on $\partial \Omega_{K}$, then we define

$$
\operatorname{ind}_{K}(T, \Omega)=\left\{k \in \mathbb{Z} \cup\{ \pm \infty\}: i_{K_{n_{j}}}\left(Q_{n_{j}} T, \Omega_{n_{j}}\right) \rightarrow k \text { for some } n_{j} \rightarrow \infty\right\} \text {, }
$$

that is, the index is the set of limit points of $i_{K_{n_{j}}}\left(Q_{n_{j}} T, \Omega_{n_{j}}\right)$, where the finite dimensional index is that defined above.

Let $L: \operatorname{dom} L \subset X \rightarrow Y$ be a Fredholm map of index zero and $P$ : $X \rightarrow X, Q: Y \rightarrow Y$ be continuous projectors such that $\operatorname{Im} P=\operatorname{Ker} L$, $\operatorname{Ker} Q=\operatorname{Im} L$ and $X=\operatorname{Ker} L \oplus \operatorname{Ker} P, Y=\operatorname{Im} L \bigoplus \operatorname{Im} Q$. The restriction of $L$ to $\operatorname{dom} L \cap \operatorname{Ker} P$, denote $L_{1}$, is a bijection onto $\operatorname{Im} L$ with continuous inverse $L_{1}^{-1}: \operatorname{Im} L \rightarrow \operatorname{dom} L \cap \operatorname{Ker} P$. Since $\operatorname{dim} \operatorname{Im} Q=\operatorname{dimKer} L$, there exists a continuous bijection $J: \operatorname{Im} Q \rightarrow \operatorname{Ker} L$. let $K$ be a cone in an infinite dimensional Banach space $X$ with projection scheme $\Gamma$. If we let $H=L+J^{-1} P$, then $H: \operatorname{dom} L \subset X \rightarrow Y$ is a linear bijection with bounded inverse. Thus $K_{1}=H(K \cap \operatorname{dom} L)$ is a cone in the Banach space $Y$. 
Let $\Omega \subset X$ be open and bounded with $\Omega_{K} \cap \operatorname{dom} L \neq \emptyset, L: \operatorname{dom} L \subset X \rightarrow Y$ a bounded Fredholm operator of index zero, $N: \bar{\Omega}_{K} \cap \operatorname{dom} L \rightarrow Y$ a bounded continuous nonlinear operator such that $L-N$ is A-proper at 0 .

We can now extend the definition of the index to A-proper maps of the form $L-N$ acting on cones.

Definition 2.4 ([4]). Let $\rho_{1}$ be a retraction from $Y$ to $K_{1}$ and assume $Q_{n} K_{1} \subset$ $K_{1}, P+J Q N+L_{1}^{-1}(I-Q) N$ maps $K \cap \operatorname{dom} L$ to $K \cap \operatorname{dom} L$ and $L x \neq N x$ on $\partial \Omega_{K}$. We define the fixed point index of $L-N$ over $\Omega_{K}$ as

$$
\operatorname{ind}_{K}([L, N], \Omega)=\operatorname{ind}_{K_{1}}(T, U),
$$

where $U=H\left(\Omega_{K}\right), T: Y \rightarrow Y$ is defined as $T y=\left(N+J^{-1} P\right) H^{-1} y$ for each $y \in Y$, and the index on the right is that of Definition 2.3.

For convenience, we recall some properties of $\operatorname{ind}_{K}$.

Proposition 2.1 ([4]). Let $L: \operatorname{dom} L \rightarrow Y$ be a Fredholm operator of index zero, $\Omega \subset X$ be open and bounded. Assume that $P+J Q N+L_{1}^{-1}(I-Q) N$ maps $K$ to $K$, and $L x \neq N x$ on $\partial \Omega_{K}$. Then we have

$\left(P_{1}\right)$ (Existence property) if $\operatorname{ind}_{K}([L, N], \Omega) \neq\{0\}$, then there exists $x \in \Omega_{K}$ such that $L x=N x$.

$\left(P_{2}\right)$ (Normality property) if $x_{0} \in \Omega_{K}$, then $\operatorname{ind}_{K}\left(\left[L,-J^{-1} P+\hat{y}_{0}\right], \Omega\right)=\{1\}$, where $\hat{y}_{0}=H x_{0}$ and $\hat{y}_{0}(y)=y_{0}$ for every $y \in H\left(\Omega_{K}\right)$.

$\left(P_{3}\right)$ (Additivity property) if $L x \neq N x$ for $x \in \bar{\Omega}_{K} \backslash\left(\Omega_{1} \cup \Omega_{2}\right)$, where $\Omega_{1}$ and $\Omega_{2}$ are disjoint relatively open subsets of $\Omega_{K}$, then

$$
\operatorname{ind}_{K}([L, N], \Omega) \subseteq \operatorname{ind}_{K}\left([L, N], \Omega_{1}\right)+\operatorname{ind}_{K}\left([L, N], \Omega_{2}\right)
$$

with equality if either of indices on the right is a singleton.

$\left(P_{4}\right)$ (Homotopy invariance property) if $L-N(\lambda, x)$ is an A-proper homotopy on $\Omega_{K}$ for $\lambda \in[0,1]$ and $\left(N(\lambda, x)+J^{-1} P\right) H^{-1}: K_{1} \rightarrow K_{1}$ and $\theta \notin(L-$ $N(\lambda, x))\left(\partial \Omega_{K}\right)$ for $\lambda \in[0,1]$, then $\operatorname{ind}_{K}([L, N(\lambda, x)], \Omega)=\operatorname{ind}_{K_{1}}\left(T_{\lambda}, U\right)$ is independent of $\lambda \in[0,1]$, where $T_{\lambda}=\left(N(\lambda, x)+J^{-1} P\right) H^{-1}$.

The following two lemmas will be used in this paper.

Lemma 2.1 ([29]). If $L: \operatorname{dom} L \rightarrow Y$ is a Fredholm operator of index zero, $\Omega$ is an open bounded set, and $\Omega_{K} \cap \operatorname{dom} L \neq \emptyset$, and let $L-\lambda N$ be A-proper for $\lambda \in[0,1]$. Assume that $N$ is bounded and $P+J Q N+L_{1}^{-1}(I-Q) N$ maps $K$ to $K$. If there exists $e \in K_{1} \backslash\{\theta\}$ such that

$$
L x-N x \neq \mu e
$$

for every $x \in \partial \Omega_{K}$ and all $\mu \geq 0$, then $\operatorname{ind}_{K}([L, N], \Omega)=\{0\}$.

We assume that there is a continuous bilinear form $[y, x]$ on $Y \times X$ such that $y \in \operatorname{Im} L$ if and only if $[y, x]=0$ for each $x \in \operatorname{Ker} L$. This condition implies that if $\left\{x_{1}, x_{2}, \ldots, x_{n}\right\}$ is a basis in $\operatorname{Ker} L$, then the linear map $J: \operatorname{Im} Q \rightarrow$ $\operatorname{Ker} L$ defined by $J y=\beta \sum_{i=1}^{n}\left[y, x_{i}\right] x_{i}, \beta \in \mathbb{R}^{+}$is an isomorphism and that 
if $y=\sum_{i=1}^{n} y_{i} x_{i}$, then $\left[J^{-1} y, x_{i}\right]=\frac{y_{i}}{\beta}$ for $1 \leq i \leq n$ and $\left[J^{-1} x_{0}, x_{0}\right]>0$ for $x_{0} \in \operatorname{Ker} L$.

Cremins [4] extended a continuation theorem related to that of Mawhin [20] and Petryshyn [26] for semilinear equations to cones refer to [4, Corollary 1] for the details. By Lemma 2.1 and [4, Corollary 1], we obtain that following existence theorem of positive solutions to a semilinear equation in cones.

Lemma 2.2. If $L: \operatorname{dom} L \rightarrow Y$ is a Fredholm operator of index zero, $K \subset X$ is a cone, $\Omega_{1}$ and $\Omega_{2}$ are open bounded sets such that $\theta \in \Omega_{1} \subset \bar{\Omega}_{1} \subset \Omega_{2}$ and $\Omega_{2} \cap K \cap \operatorname{dom} L \neq \emptyset$, where $\theta$ is the zero element of $X$. Suppose that $L-\lambda N$ is A-proper for $\lambda \in[0,1]$ with $N: \overline{\Omega_{2} \cap K} \rightarrow Y$ is bounded. Assume that

$\left(C_{1}\right)(P+J Q N)(K) \subset K$ and $\left(P+J Q N+L_{1}^{-1}(I-Q) N\right)(K) \subset K$,

$\left(C_{2}\right) L x \neq \lambda N x$ for $x \in \partial \Omega_{2} \cap K, \lambda \in(0,1]$,

$\left(C_{3}\right) Q N x \neq 0$ for $x \in \partial \Omega_{2} \cap K \cap \operatorname{Ker} L$,

$\left(C_{4}\right)[Q N x, x] \leq 0$ for all $x \in \partial \Omega_{2} \cap K \cap \operatorname{Ker} L$,

$\left(C_{5}\right)$ there exists $e \in K_{1} \backslash\{\theta\}$ such that

$$
L x-N x \neq \mu e \text { for every } \mu \geq 0, x \in \partial \Omega_{1} \cap K .
$$

Then there exists $x \in \operatorname{dom} L \cap K \cap\left(\bar{\Omega}_{2} \backslash \Omega_{1}\right)$ such that $L x=N x$.

Remark 2.1. It is worth mentioning that the positive or nonnegative solutions of an operator equation $L x=N x$ was also discussed by a recent paper of O'Regan and Zima [24] and the earlier papers [5, 7, 23, 27, 28].

\section{Main results}

The goal of this section is to apply Lemma 2.2 to discuss the existence and multiplicity of positive solutions for the BVP (1.1). For simplicity of notation, we denote $\xi_{0}=0, \xi_{m-1}=1, \alpha_{0}=\alpha_{m-1}=\beta_{0}=\beta_{m-1}=0$ and the function $l(s), s \in[0,1]$ as follow

$$
\begin{gathered}
l(s)=1-s+\frac{\sum_{i=0}^{m-1} \beta_{i} \xi_{i}-1}{\sum_{i=0}^{m-1} \alpha_{i} \xi_{i}} \sum_{i=k}^{m-1} \alpha_{i}\left(\xi_{i}-s\right)-\sum_{i=k}^{m-1} \beta_{i}\left(\xi_{i}-s\right), \\
\xi_{k-1} \leq s \leq \xi_{k}, k=1,2, \ldots, m-1 .
\end{gathered}
$$

It is easy to check that $l(s) \geq 0, s \in[0,1]$. Denote the function $G(t, s)$ as follow:

$$
G(t, s)=\left\{\begin{array}{l}
\frac{(1-s)^{2}}{2}+\frac{3 t^{2}+5}{6 \int_{0}^{1} l(s) d s} l(s), \quad 0 \leq t \leq s \leq 1 \\
\frac{(1-s)^{2}}{2}+s-t+\frac{3 t^{2}+5}{6 \int_{0}^{1} l(s) d s} l(s), \quad 0 \leq s \leq t \leq 1 .
\end{array}\right.
$$


Note that $G(t, s) \geq 0, t, s \in[0,1]$. We also set

$$
\kappa=\min \left\{\min _{s \in[0,1]} \frac{\int_{0}^{1} l(s) d s}{l(s)}, \frac{1}{\max _{t, s \in[0,1]} G(t, s)}\right\} .
$$

Note that $\kappa<1$.

Consider the Banach spaces

$$
X=Y=C[0,1]
$$

with

$$
\|x\|=\max _{t \in[0,1]}|x(t)| .
$$

Let $L: \operatorname{dom} L \rightarrow Y$ and $N: X \rightarrow Y$ with

$$
\operatorname{dom} L=\left\{x \in X: x^{\prime \prime} \in C[0,1], x(0)=\sum_{i=1}^{m-2} \alpha_{i} x\left(\xi_{i}\right)\right\}
$$

be given by $(L x)(t)=-x^{\prime \prime}(t)$ and $(N x)(t)=f(t, x(t))$ for $t \in[0,1]$.

It is easy to check that

$$
\begin{aligned}
& \operatorname{Ker} L=\{x \in \operatorname{dom} L: x(t) \equiv c \text { on }[0,1], c \in \mathbb{R}\}, \\
& \operatorname{Im} L=\left\{y \in Y: \int_{0}^{1} l(s) y(s) d s=0\right\}, \\
& \operatorname{dim} \operatorname{Ker} L=\operatorname{codim} \operatorname{Im} L=1,
\end{aligned}
$$

so that $L$ is a Fredholm operator of index zero.

Next, define the projections $P: X \rightarrow X$ by

$$
P x=\int_{0}^{1} x(s) d s,
$$

and $Q: Y \rightarrow Y$ by

$$
Q y=\frac{1}{\int_{0}^{1} l(s) d s} \int_{0}^{1} l(s) y(s) d s .
$$

Furthermore, we define the isomorphism $J: \operatorname{Im} Q \rightarrow \operatorname{Im} P$ as $J y=\beta y$, where $\beta=1$. We are easy to verify that the inverse operator $L_{1}^{-1}: \operatorname{Im} L \rightarrow \operatorname{dom} L \cap$ $\operatorname{Ker} P$ of $\left.L\right|_{\operatorname{dom} L \cap \operatorname{Ker} P}: \operatorname{dom} L \cap \operatorname{Ker} P \rightarrow \operatorname{Im} L$ is $\left(L_{1}^{-1} y\right)(t)=\int_{0}^{1} k(t, s) y(s) d s$, where

$$
k(t, s)= \begin{cases}\frac{(1-s)^{2}}{2}, & 0 \leq t \leq s \leq 1 \\ \frac{(1-s)^{2}}{2}+s-t, & 0 \leq s \leq t \leq 1\end{cases}
$$

Now we can state and prove our main results.

Theorem 3.1. Assume that there exist two positive numbers $a, b$ such that $\left(H_{1}\right) f(t, x) \geq-\kappa x$ for all $t \in[0,1], x \geq 0$.

$\left(\mathrm{H}_{2}\right)$ If one of the two conditions 
(i) $\min _{t \in[0,1]} f(t, a)>0, \max _{t \in[0,1]} f(t, b)<0$

and

(ii) $\max _{t \in[0,1]} f(t, a)<0, \min _{t \in[0,1]} f(t, b)>0$

is satisfied, then the BVP (1.1) has at least one positive solution $x^{*} \in X$ satisfying $\min \{a, b\} \leq\left\|x^{*}\right\|_{X} \leq \max \{a, b\}$.

Proof. Consider the cone

$$
K=\{x \in X: x(t) \geq 0, t \in[0,1]\} .
$$

It is easy to see $a \neq b$. Without loss of generality, let $a<b$.

First, we note that $L$, as so defined, is a Fredholm operator of index zero, $L_{1}^{-1}$ is compact by Arzela-Ascoli theorem and thus $L-\lambda N$ is A-proper for $\lambda \in[0,1]$ by (a) of Lemma 2 in [26].

For each $x \in K$, then by condition $\left(H_{1}\right)$ that

$$
\begin{gathered}
P x+J Q N x \\
=\int_{0}^{1} x(s) d s+\frac{1}{\int_{0}^{1} l(s) d s} \int_{0}^{1} l(s) f(s, x(s)) d s \\
\geq \int_{0}^{1}\left(1-\frac{\kappa}{\int_{0}^{1} l(s) d s} l(s)\right) x(s) d s \\
\geq 0, \\
P x+J Q N x+L_{1}^{-1}(I-Q) N x \\
=\int_{0}^{1} x(s) d s+\frac{1}{\int_{0}^{1} l(s) d s} \int_{0}^{1} l(s) f(s, x(s)) d s \\
+\int_{0}^{1} k(t, s)\left[f(s, x(s))-\frac{1}{\int_{0}^{1} l(s) d s} \int_{0}^{1} l(\tau) f(\tau, x(\tau)) d \tau\right] d s \\
=\int_{0}^{1} x(s) d s+\int_{0}^{1} G(t, s) f(s, x(s)) d s \\
\geq \int_{0}^{1}(1-\kappa G(t, s)) x(s) d s \geq 0 .
\end{gathered}
$$

This implies that condition $\left(C_{1}\right)$ of Lemma 2.2 is satisfied. To apply Lemma 2.2 , we should define two open bounded subsets $\Omega_{1}, \Omega_{2}$ of $X$ so that $\left(C_{2}\right)-\left(C_{5}\right)$ of Lemma 2.2 hold.

We prove only Case $\left(H_{2}\right)$ (i). In the same way, we can prove the case $\left(H_{2}\right)(i i)$. Let

$$
\Omega_{1}=\left\{x \in X:\|x\|_{X}<a\right\}, \Omega_{2}=\left\{x \in X:\|x\|_{X}<b\right\} .
$$

Clearly, $\Omega_{1}$ and $\Omega_{2}$ are bounded and open sets and

$$
\theta \in \Omega_{1} \subset \bar{\Omega}_{1} \subset \Omega_{2} .
$$


Next we show that $\left(H_{2}\right)(\mathrm{i})$ implies $\left(C_{2}\right)$. That is, suppose that there exist $x_{1} \in K \cap \partial \Omega_{2} \cap \operatorname{dom} L$ and $\lambda_{1} \in(0,1]$ such that $L x_{1}=\lambda_{1} N x_{1}$ then $x_{1}^{\prime \prime}(t)=-\lambda_{1} f\left(t, x_{1}(t)\right)$ for all $t \in[0,1]$. Let $t_{1} \in[0,1]$, such that $x_{1}\left(t_{1}\right)=$ $\max _{t \in[0,1]} x_{1}(t)=b$. From boundary conditions, we have $t_{1} \in(0,1)$. In this case, $x_{1}^{\prime}\left(t_{1}\right)=0, x_{1}^{\prime \prime}\left(t_{1}\right) \leq 0$. This gives

$$
0 \geq x_{1}^{\prime \prime}\left(t_{1}\right)=-\lambda_{1} f\left(t_{1}, x\left(t_{1}\right)\right)=-\lambda_{1} f\left(t_{1}, b\right)>0,
$$

which contradicts $\left(H_{2}\right)(\mathrm{i})$. So for each $x \in \partial \Omega_{2} \cap K \cap \operatorname{dom} L$ and $\lambda \in(0,1]$, we have $L x \neq \lambda N x$. Thus $\left(C_{2}\right)$ of Lemma 2.2 is satisfied.

To prove $\left(C_{4}\right)$ of Lemma 2.2 , we define the bilinear form $[\cdot, \cdot]: Y \times X \rightarrow \mathbb{R}$ as

$$
[y, x]=\int_{0}^{1} l(s) y(s) x(s) d s .
$$

It is clear that $[\cdot, \cdot]$ is continuous and satisfies $[y, x]=0$ for every $x \in \operatorname{Ker} L, y \in$ $\operatorname{Im} L$. In fact, for any $x \in \operatorname{Ker} L$ and $y \in \operatorname{Im} L$, we have $x \equiv c$, a constant, and there exists $x \in X$ such that $y(s)=-x^{\prime \prime}(s)$ for each $s \in[0,1]$. By $x(0)=\sum_{i=1}^{m-2} \alpha_{i} x\left(\xi_{i}\right), x(1)=\sum_{i=1}^{m-2} \beta_{i} x\left(\xi_{i}\right)$, we get

$$
[y, x]=\int_{0}^{1} l(s) y(s) x(s) d s=-c \int_{0}^{1} l(s) x^{\prime \prime}(s) d s=0 .
$$

Let $x \in \operatorname{Ker} L \cap \partial \Omega_{2} \cap K$. Then $x(t) \equiv b$, so we have by condition $\left(H_{2}\right)(\mathrm{i})$

$$
\begin{aligned}
Q N x & =\frac{1}{\int_{0}^{1} l(s) d s} \int_{0}^{1} l(s) f(s, b) d s \neq 0 \\
{[Q N x, x] } & =\int_{0}^{1} l(s)\left(\frac{1}{\int_{0}^{1} l(s) d s} \int_{0}^{1} l(s) f(s, b) d s \cdot b\right) d s \\
& =b \cdot \int_{0}^{1} l(s) d s \cdot \frac{1}{\int_{0}^{1} l(s) d s} \int_{0}^{1} l(s) f(s, b) d s \\
& =b \cdot \int_{0}^{1} l(s) f(s, b) d s \\
& <0 .
\end{aligned}
$$

Thus $\left(C_{3}\right)$ and $\left(C_{4}\right)$ of Lemma 2.2 are verified.

Finally, we prove $\left(C_{5}\right)$ of Lemma 2.2 is satisfied. We may suppose that $L x \neq N x, \forall x \in \partial \Omega_{1} \cap K \cap \operatorname{dom} L$. Otherwise, the proof is completed. Let $e_{*} \equiv 1 \in K_{1} \backslash\{\theta\}$. We claim that

$$
L x-N x \neq \mu e_{*}, \forall x \in \partial \Omega_{1} \cap K \cap \operatorname{dom} L, \mu \geq 0 .
$$

In fact, if not, there exist $x_{2} \in \partial \Omega_{1} \cap K \cap \operatorname{dom} L, \mu_{1}>0$, such that

$$
L x_{2}-N x_{2}=\mu_{1} \text {. }
$$

Since $Q L=\theta$, operating on both sides of the latter equation by $Q$, we obtain

$$
Q N x_{2}+Q \mu_{1}=0 \text {, }
$$


that is

$$
\frac{1}{\int_{0}^{1} l(s) d s} \int_{0}^{1} l(s)\left(f\left(s, x_{2}(s)\right)+\mu_{1}\right) d s=0 .
$$

For any $x_{2} \in \partial \Omega_{1} \cap K \cap \operatorname{dom} L$, we have $\left\|x_{2}\right\|_{X}=a$. There exists $t_{2} \in[0,1]$, such that $x_{2}\left(t_{2}\right)=a$. By condition $\left(H_{2}\right)(\mathrm{i})$ and $\mu_{1}>0$,

$$
\frac{1}{\int_{0}^{1} l(s) d s} \int_{0}^{1} l(s)\left(f\left(s, x_{2}(s)\right)+\mu_{1}\right) d s=\frac{1}{\int_{0}^{1} l(s) d s} \int_{0}^{1} l(s)\left(f(s, a)+\mu_{1}\right) d s>0,
$$

in contradiction to (3.3). So (3.2) holds, that is $\left(C_{5}\right)$ of Lemma 2.2 is verified.

Thus all conditions of Lemma 2.2 are satisfied and there exists $x^{*} \in K \cap$ $\left(\bar{\Omega}_{2} \backslash \Omega_{1}\right) \cap \operatorname{dom} L$ such that $L x^{*}=N x^{*}$ and the assertion follows. Thus $x^{*} \in K$ and $a \leq\left\|x^{*}\right\|_{X} \leq b$.

Let $[c]$ be the integer part of $c$. The following result concerns the existence results of $n$ positive solutions.

Theorem 3.2. Assume that there exist $n+1$ positive numbers $a_{1}<a_{2}<\cdots<$ $a_{n+1}$ such that

$\left(H_{1}\right)^{\prime} f(t, x) \geq-\kappa x$ for all $t \in[0,1], x \geq 0$.

$\left(\mathrm{H}_{2}\right)^{\prime}$ If one of the two conditions

(i) $\min _{t \in[0,1]} f\left(t, a_{2 i-1}\right)>0, i=1,2, \ldots,\left[\frac{n+2}{2}\right]$, $\max _{t \in[0,1]} f\left(t, a_{2 i}\right)<0, i=1,2, \ldots,\left[\frac{n+1}{2}\right]$

and

(ii) $\max _{t \in[0,1]} f\left(t, a_{2 i-1}\right)<0, i=1,2, \ldots,\left[\frac{n+2}{2}\right]$,

$$
\min _{t \in[0,1]} f\left(t, a_{2 i}\right)>0, i=1,2, \ldots,\left[\frac{n+1}{2}\right]
$$

is satisfied, then the $B V P(1.1)$ has at least one positive solutions $x_{i}^{*} \in K$, $i=1,2, \ldots, n$ satisfying $a_{i}<\left\|x_{i}^{*}\right\|_{X}<a_{i+1}$.

Proof. Modeling the proof of Theorem 3.1, we can prove that if there exist two positive numbers $a, b$ such that $\min _{t \in[0,1]} f(t, a)>0$ and $\max _{t \in[0,1]} f(t, b)<0$, then BVP (1.1) has at least one positive solution $x^{*} \in K$ satisfying $\min \{a, b\}<$ $\left\|x^{*}\right\|_{X}<\max \{a, b\}$.

By the claim, for every pair of positive numbers $\left\{a_{i}, a_{i+1}\right\}, i=1,2, \ldots, n$, (1.1) has at least one positive solutions $x_{i}^{*} \in K$ satisfying $a_{i}<\left\|x_{i}^{*}\right\|_{X}<$ $a_{i+1}$.

We have the following existence result for two positive solutions.

Corollary 3.1. Assume that there exist three positive numbers $a_{1}<a_{2}<a_{3}$ such that

$\left(H_{1}\right)^{\prime \prime} f(t, x) \geq-\kappa x$ for all $t \in[0,1], x \geq 0$.

$\left(\mathrm{H}_{2}\right)^{\prime \prime}$ If one of the two conditions 
(i) $\min _{t \in[0,1]} f\left(t, a_{1}\right)>0, \max _{t \in[0,1]} f\left(t, a_{2}\right)<0, \min _{t \in[0,1]} f\left(t, a_{3}\right)>0$

(ii) $\max _{t \in[0,1]} f\left(t, a_{1}\right)<0, \min _{t \in[0,1]} f\left(t, a_{2}\right)>0, \max _{t \in[0,1]} f(t, x)<0$ is satisfied, then the $B V P(1.1)$ has at least two positive solutions $x_{1}^{*}, x_{2}^{*} \in K$ satisfying $a_{1} \leq\left\|x_{1}^{*}\right\|_{X}<a_{2}<\left\|x_{2}^{*}\right\|_{X} \leq a_{3}$.

We also have the following existence result for three positive solutions.

Corollary 3.2. Assume that there exist four positive numbers $a_{1}<a_{2}<a_{3}<$ $a_{4}$ such that

$\left(H_{1}\right)^{\prime \prime \prime} f(t, x) \geq-\kappa x$ for all $t \in[0,1], x \geq 0$.

$\left(\mathrm{H}_{2}\right)^{\prime \prime \prime}$ If one of the two conditions

(i) $\min _{t \in[0,1]} f\left(t, a_{1}\right)>0, \max _{t \in[0,1]} f\left(t, a_{2}\right)<0, \min _{t \in[0,1]} f\left(t, a_{3}\right)>0, \max _{t \in[0,1]} f\left(t, a_{4}\right)<0$ and

(ii) $\max _{t \in[0,1]} f\left(t, a_{1}\right)<0, \min _{t \in[0,1]} f\left(t, a_{2}\right)>0, \max _{t \in[0,1]} f\left(t, a_{3}\right)<0, \min _{t \in[0,1]} f\left(t, a_{4}\right)>0$ is satisfied, then the BVP (1.1) has at least three positive solutions $x_{1}^{*}, x_{2}^{*}, x_{3}^{*} \in$ $K$ satisfying $a_{1} \leq\left\|x_{1}^{*}\right\|_{X}<a_{2}<\left\|x_{2}^{*}\right\|_{X}<a_{3}<\left\|x_{3}^{*}\right\|_{X} \leq a_{4}$.

Remark 3.1. In this paper, we give some multiplicity results for positive solutions. Up to now, there were fewer papers that considered the existence of multiple positive solutions for multi-point boundary value problem at resonance. Therefore, the results of this paper is new.

\section{Example}

In this section, we give an example to illustrate the main results of the paper. Consider the following second-order three-point boundary value problem (BVP)

$$
\left\{\begin{array}{l}
-x^{\prime \prime}(t)=-\frac{433(5 t+2)}{9200} \sin x, \quad 0<t<1, \\
x(0)=\frac{1}{2} x\left(\frac{1}{4}\right)+\frac{1}{2} x\left(\frac{1}{2}\right), \quad x(1)=\frac{1}{3} x\left(\frac{1}{4}\right)+\frac{2}{3} x\left(\frac{1}{2}\right),
\end{array}\right.
$$

Corresponding to problem (1.1), we have $\alpha_{1}=\alpha_{2}=\frac{1}{2}, \beta_{1}=\frac{1}{3}, \beta_{2}=\frac{2}{3}, \xi_{1}=$ $\frac{1}{4}, \xi_{2}=\frac{1}{2}$. By calculating directly, we have $\kappa=\frac{433}{920}$. It is easy to check that $-\frac{433(5 t+2)}{9200} \sin x \geq-\kappa x, x \geq 0, t \in[0,1]$.

(1) We take $a=\frac{\pi}{2}, b=\frac{3 \pi}{2}$, then all the conditions of Theorem 3.1 are satisfied. Thus BVP (4.1) has at least one positive solution $x^{*}$ satisfying $\frac{\pi}{2} \leq$ $\left\|x^{*}\right\|_{X} \leq \frac{3 \pi}{2}$.

(2) We take $a_{1}=\frac{\pi}{2}, a_{2}=\frac{3 \pi}{2}, a_{3}=\frac{5 \pi}{2}$, then all the conditions of Corollary 3.1 are satisfied. Thus BVP $(4.1)$ has at least two positive solutions $x_{1}^{*}, x_{2}^{*}$ satisfying $\frac{\pi}{2} \leq\left\|x_{1}^{*}\right\|_{X}<\frac{3 \pi}{2}<\left\|x_{2}^{*}\right\|_{X} \leq \frac{5 \pi}{2}$.

(3) We take $a_{1}=\frac{\pi}{2}, a_{2}=\frac{3 \pi}{2}, a_{3}=\frac{5 \pi}{2}, a_{4}=\frac{7 \pi}{2}$, then all the conditions of Corollary 3.2 are satisfied. Thus BVP (4.1) has at least three positive solutions $x_{1}^{*}, x_{2}^{*}, x_{3}^{*}$ satisfying $\frac{\pi}{2} \leq\left\|x_{1}^{*}\right\|_{X}<\frac{3 \pi}{2}<\left\|x_{2}^{*}\right\|_{X}<\frac{5 \pi}{2}<\left\|x_{3}^{*}\right\|_{X} \leq \frac{7 \pi}{2}$.

(4) We take $a_{2 i-1}=\frac{(4 i-3) \pi}{2}, i=1,2, \ldots,\left[\frac{n+2}{2}\right] ; a_{2 i}=\frac{(4 i-1) \pi}{2}, i=1,2, \ldots$, $\left[\frac{n+1}{2}\right]$, then all the conditions of Theorem 3.2 are satisfied. Thus BVP (4.1) has 
at least $n$ positive solutions $x_{i}^{*}, i=1,2, \ldots, n$ satisfying $a_{i}<\left\|x_{i}^{*}\right\|_{X}<a_{i+1}$, $i=1,2, \ldots, n$.

\section{References}

[1] C. Bai and J. Fang, Existence of positive solutions for three-point boundary value problems at resonance, J. Math. Anal. Appl. 291 (2004), no. 2, 538-549.

[2] A. V. Bitsadze, On the theory of nonlocal boundary value problems, Soviet Math. Dokl. 30 (1984), 8-10.

[3] A. V. Bitsadze and A. A. Samarskii, On a class of conditionally solvable nonlocal boundary value problems for harmonic functions, Soviet Math. Dokl. 31 (1985), 91-94.

[4] C. T. Cremins, A fixed-point index and existence theorems for semilinear equations in cones, Nonlinear Anal. 46 (2001), no. 6, 789-806.

[5] M. Feckan, Existence of nonzero nonnegative solutions of semilinear equations at resonance, Comment. Math. Univ. Carolin. 39 (1998), no. 4, 709719.

[6] W. Feng and J. R. L. Webb, Solvability of three-point boundary value problems at resonance, Nonlinear Anal. 30 (1997), no. 6, 3227-3238.

[7] R. E. Gaines and J. Santanilla, A coincidence theorem in convex sets with applications to periodic solutions of ordinary differential equations, Rocky Mountain J. Math. 12 (1982), no. 4, 669-678.

[8] C. P. Gupta, Existence theorems for a second order $m$-point boundary value problem at resonance, Internat. J. Math. Math. Sci. 18 (1995), no. 4, 705-710.

[9] Solvability of a multi-point boundary value problem at resonance, Results Math. 28 (1995), no. 3-4, 270-276.

[10] - A note on a second order three point boundary value problem, J. Math. Anal. Appl. 186 (1994), no. 1, 277-281.

[11] C. P. Gupta, S. K. Ntouyas, and P. C. Tsamatos, On an m-point boundary value problem for second order ordinary differential equations, Nonlinear Anal. 23 (1994), no. 11, 14271436.

[12] X. Han, Positive solutions for a three-point boundary value problem at resonance, J. Math. Anal. Appl. 336 (2007), no. 1, 556-568.

[13] V. A. Il'in and E. I. Moiseev, Nonlocal boundary value problems of the second kind for the Sturm-Liouville operator, J. Differential Equations 23 (1987), 979-987.

[14] G. Infante and M. Zima, Positive solutions of multi-point boundary value problems at resonance, Nonlinear Anal. 69 (2008), no. 8, 2458-2465.

[15] K. Q. Lan, Properties of kernels and eigenvalues for three point boundary value problems, Discrete Contin. Dyn. Syst. (Suppl.) (2005), 546-555.

[16] S. Liang and L. Mu, Multiplicity of positive solutions for singular three-point boundary value problems at resonance, Nonlinear Anal. 71 (2009), no. 7-8, 2497-2505.

[17] R. Ma, Existence theorems for a second order m-point boundary value problem, J. Math. Anal. Appl. 211 (1997), no. 2, 545-555.

[18] _ Existence of positive solutions for superlinear semipositone m-point boundaryvalue problems, Proc. Edinb. Math. Soc. (2) 46 (2003), no. 2, 279-292.

[19] Existence results of a m-point boundary value problem at resonance, J. Math. Anal. Appl. 294 (2004), no. 1, 147-157.

[20] J. Mawhin, Landesman-Lazer's type problems for nonlinear equations, Confer. Sem. Mat. Univ. Bari No. 147 (1977), 22 pp.

[21] _ Topological degree methods in nonlinear boundary value problems, CBMS Regional Conference Series in Mathematics, 40, American Mathematical Society, Providence, RI, 1979. 
[22] _ Topological degree and boundary value problems for nonlinear differential equations, Topological methods for ordinary differential equations (Montecatini Terme, 1991), 74142, Lecture Notes in Math., 1537, Springer, Berlin, 1993.

[23] J. Nieto, Existence of solutions in a cone for nonlinear alternative problems, Proc. Amer. Math. Soc. 94 (1985), no. 3, 433-436.

[24] D. O'Regan and M. Zima, Leggett-Williams norm-type theorems for coincidences, Arch. Math. (Basel) 87 (2006), no. 3, 233-244.

[25] P. K. Palamides, Multi-point boundary-value problems at resonance for nth-order differential equations: Positive and monotone solutions, Electron. J. Differential Equations 2004 (2004), no. 25, 1-14.

[26] W. V. Petryshyn, Using degree theory for densely defined A-proper maps in the solvability of semilinear equations with unbounded and noninvertible linear part, Nonlinear Anal. 4 (1980), no. 2, 259-281.

[27] B. Przeradzki, A note on solutions of semilinear equations at resonance in a cone, Ann. Polon. Math. 58 (1993), no. 1, 95-103.

[28] J. Santanilla, Existence of nonnegative solutions of a semilinear equation at resonance with linear growth, Proc. Amer. Math. Soc. 105 (1989), no. 4, 963-971.

[29] F. Wang and F. Zhang, Some new approach to the computation for fixed point index and applications, Bull. Malays. Math. Sci. Soc. in press.

[30] J. R. L. Webb, Positive solutions of some three point boundary value problems via fixed point index theory, Nonlinear Anal. 47 (2001), no. 7, 4319-4332.

[31] _ Multiple positive solutions of some nonlinear heat flow problems, Discrete Contin. Dyn. Syst. (Suppl.) (2005), 895-903.

[32] J. R. L. Webb and M. Zima, Multiple positive solutions of resonant and non-resonant nonlocal boundary value problems, Nonlinear Anal. 71 (2009), no. 3-4, 1369-1378.

[33] X. Xu, Positive solutions for singular m-point boundary value problems with positive parameter, J. Math. Anal. Appl. 291 (2004), no. 1, 352-367.

[34] _ Multiplicity results for positive solutions of some semi-positone three-point boundary value problems, J. Math. Anal. Appl. 291 (2004), no. 2, 673-689.

[35] A. Yang and W. Ge, Positive solutions of self-adjoint boundary value problem with integral boundary conditions at resonance, J. Korea Soc. Math. Educ. Ser. B: Pure Appl. Math. 15 (2008), no. 4, 407-414.

[36] L. Yang and C. Shen, On the existence of positive solution for a kind of multi-point boundary value problem at resonance, Nonlinear Anal. 72 (2010), no. 11, 4211-4220.

[37] G. Zhang and J. Sun, Positive solutions of m-point boundary value problems, J. Math. Anal. Appl. 291 (2004), no. 2, 406-418.

[38] Q. Zhang and D. Jiang, Upper and lower solutions method and a second order three-point singular boundary value problem, Comput. Math. Appl. 56 (2008), no. 4, 1059-1070.

[39] M. Zima, Existence of positive solutions for a second-order three-point boundary value problem at resonance, Dynamic systems and applications. Vol. 5, 52732, Dynamic, Atlanta, GA, 2008.

FENG WANG

School of Mathematics and Physics

Changzhou University

Changzhou, Jiangsu 213164, P. R. China

AND

Department of Mathematics

College of SCIEnce

HoHAi University

NAnjing, Jiangsu 210098, P. R. China

E-mail address: fengwang188@163.com 
FANG ZHANG

School of Mathematics and Physics

Changzhou University

Changzhou, Jiangsu 213164, P. R. China

E-mail address: fangzhang188@163.com 